\title{
Radiation Effects on Free Convection MHD Couette Flow Started Exponentially with Variable Wall Temperature in Presence of Heat Generation
}

\author{
Sanatan Das, Bhaskar Chandra Sarkar, Rabindra Nath Jana \\ Department of Applied Mathematics, Vidyasagar University, Midnapore, India \\ Email: jana261171@yahoo.co.in
}

Received December 29, 2011; revised January 29, 2012; accepted February 5, 2012

\begin{abstract}
Radiation effects on free convection MHD Couette flow started exponentially with variable wall temperature in the presence of heat generation have been studied. The governing equations are solved analytically using the Laplace transform technique. The variations of velocity and fluid temperature are presented graphically. It is observed that the velocity decreases with an increase in either magnetic parameter or radiation parameter or Prandtl number. It is also observed that the velocity increases with an increase in either heat generation parameter or Grashof number or accelerated parameter or time. An increase in either radiation parameter or Prandtl number leads to fall in the fluid temperature. It is seen that the fluid temperature increases with an increase in either heat generation parameter or time. Further, it is seen that the shear stress at the moving plate decreases with an increase in either magnetic parameter or radiation parameter while it increases with an increase in either heat generation parameter or Prandtl number. The rate of heat transfer increases with an increase in either Prandtl number or time whereas it decreases with an increase in heat generation parameter.
\end{abstract}

Keywords: MHD Couette Flow; Free Convection; Magnetic Parameter; Radiation; Heat Generation; Prandtl Number; Grashof Number and Accelerated Parameter

\section{Introduction}

Couette flow is one of the basic flow in fluid dynamics that refers to the laminar flow of a viscous fluid in the space between two parallel plates, one of which is moving relative to the other. The flow is driven by virtue of viscous drag force acting on the fluid and the applied pressure gradient parallel to the plates. Couette flow is frequently used in physics and engineering to illustrate shear-driven fluid motion. Some important application areas of Couette motion are MHD power generators and pumps, aerodynamics heating, electrostatic precipitation, polymer technology, petroleum industry, purification of crude oil etc. In space technology applications and at higher operating temperatures, radiation effects can be quite significant. Radiative free convection MHD Couette flows are frequently encountered in many scientific and environmental processes, such as astrophysical flows, heating and cooling of chambers and solar power technology. Heat transfer by simultaneous radiation and convection has applications in numerous technological problems including combustion, furnace design, the design of high temperature gas cooled nuclear reactors, nuclear reactor safety, fluidized bed heat exchanger, fire spreads, solar fans, solar collectors natural convection in cavities, turbid water bodies, photo chemical reactors and many others. Free convection in channels formed by vertical plates has received attention among the researchers in last few decades due to it's widespread importance in engineering applications like cooling of electronic equipments, design of passive solar systems for energy conversion, design of heat exchangers, human comfort in buildings, thermal regulation processes and many more. Researchers in this field such as Singh [1], Singh et al. [2], Jha et al. [3], Joshi [4], Miyatake et al. [5], Tanaka et al. [6], Mohanty [7]. Jha [8] have studied the natural Convection in unsteady MHD Couette flow. The radiative heat transfer to magnetohydrodynamic Couette flow with variable wall temperature have been investigated by Ogulu and Motsa [9]. Chaudhary and Jain [10] have analyzed the exact solutions of incompressible Couette flow with constant temperature and constant heat flux on walls in the presence of radiation. The radiation effects on MHD Couette flow with heat transfer between two parallel plates have been examined by Mebine [11]. Jha and Ajibade [12] have discussed the free convective flow 
of heat generating fluid between vertical porous plates with periodic heat input. Jha and Ajibade [13] have studied the unsteady free convective Couette flow of heat generating/absorbing fluid. MHD oscillatory Couette flow of a radiating viscous fluid in a porous medium with periodic wall temperature have been investigated by IsraelCookey et al. [14]. The effects of thermal radiation and free convection currents on the unsteady Couette flow between two vertical parallel plates with constant heat flux at one boundary have been studied by Narahari [15]. Unsteady free convective Couette flow of heat generating/absorbing fluid in porous medium has been investigated by Deka and Bhattacharya [16]. Kumar and Varma [17] have studied the radiation effects on MHD flow past an impulsively started exponentially accelerated vertical plate with variable temperature in the presence of heat generation.

In this present paper, we have investigated the radiation effects on free convection MHD Couette flow of a viscous incompressible heat generating fluid in the presence of variable temperature. It is observed that the velocity $u_{1}$ decreases with an increase in either magnetic parameter $M^{2}$ or radiation parameter $R$ or Prandtl number $\mathrm{Pr}$. It is also observed that the velocity $u_{1}$ increases with an increase in either heat generation parameter or Grashof number $\mathrm{Gr}$ or accelerated parameter $a$ or time $\tau$. An increase in either radiation parameter $R$ or Prandtl number $\operatorname{Pr}$ leads to fall in the fluid temperature $\theta$. It is seen that the fluid temperature $\theta$ increases with an increase in either heat generation parameter $\phi$ or time $\tau$. Further, it is seen that the shear stress at the moving plate $\tau_{x}$ decreases with an increase in either magnetic parameter or radiation parameter while it increases with an increase in either heat generation parameter or Prandtl number. The rate of heat transfer $-\theta^{\prime}(0)$ increases with an increase in either Prandtl number Pr or time $\tau$ whereas it decreases with an increase in heat generation parameter $\phi$.

\section{Formulation of the Problem and Its Solutions}

Consider the unsteady free convection MHD Couette flow of a viscous incompressible radiative heat generating fluid between two infinite vertical parallel walls separated by a distance $h$. The flow is set up by the buoyancy force arising from the temperature gradient occurring as a result of asymmetric heating of the parallel plates as well as constant motion of one of the plates. Choose a cartesian co-ordinates system with the $x$-axis along one of the plates in the vertically upward direction and the $y$-axis normal to the plates (See Figure 1). Initially, at time $t \leq 0$, both the plates and the fluid are assumed to be at the same temperature $T_{h}$ and station-

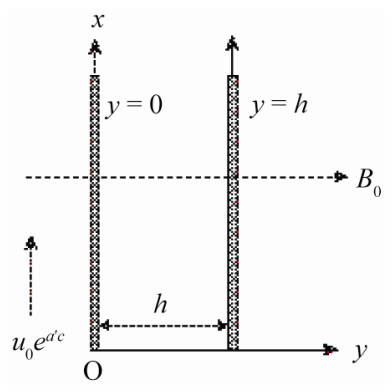

Figure 1. Geometry of the problem

ary. At time $t>0$, the plate at $y=0$ starts moving in its own plane with a velocity $u_{0} e^{a^{\prime} t}$ and is heated with temperature $T_{h}+\left(T_{0}-T_{h}\right) \frac{t}{t_{0}}$ whereas the plate at

$y=h$ is stationary and maintained at a constant temperature $T_{h}$, where $u_{0}$ and $a^{\prime}$ are constants. A uniform magnetic field of strength $B_{0}$ is imposed perpendicular to the plates. It is also assumed that the radiative heat flux in the $x$-direction is negligible as compared to that in the $y$-direction. As the plates are infinitely long, the velocity and temperature fields are functions of $y$ and $t$ only.

The Boussinesq approximation is assumed to hold and for the evaluation of the gravitational body force, the density is assumed to depend on the temperature according to the equation of state

$$
\rho=\rho_{0}\left[1-\beta\left(T_{0}-T_{h}\right)\right],
$$

where $T$ is the fluid temperature, $\rho$ the fluid density, $\beta$ the coefficient of thermal expansion and $\rho_{0}$ the density at the entrance of the channel.

For the fully developed flow, the governing equations are

$$
\begin{aligned}
& \frac{\partial u}{\partial t}=-\frac{1}{\rho} \frac{\partial p}{\partial x}+g \beta\left(T-T_{h}\right)+v \frac{\partial^{2} u}{\partial y^{2}}-\frac{\sigma B_{0}^{2}}{\rho} u, \\
& \rho c_{p} \frac{\partial T}{\partial t}=k \frac{\partial^{2} T}{\partial y^{2}}-\frac{\partial q_{r}}{\partial y}+Q_{0}\left(T-T_{h}\right),
\end{aligned}
$$

where $u$ is the velocity in the $x$-direction, $g$ the acceleration due to gravity, $v$ the kinematic coefficient of viscosity, $k$ the thermal conductivity, $c_{p}$ the specific heat at constant pressure, $q_{r}$ the radiative heat flux and $Q_{0}$ a constant.

The initial and the boundary conditions for velocity and temperature distribution are as follows:

$$
\begin{array}{ll}
u=0, & T=T_{h} \text { for } 0 \leq y \leq h \text { and } t \leq 0, \\
u=u_{0} e^{a^{\prime} t}, & T=T_{h}+\left(T_{0}-T_{h}\right) \frac{t}{t_{0}} \text { at } y=0 \text { for } t>0, \\
u=0, & T=T_{h} \text { at } y=h \text { for } t>0 .
\end{array}
$$

It has been shown by Cogley et al. [13] that in the 
optically thin limit for a non-gray gas near equilibrium, the following relation holds

$$
\frac{\partial q_{r}}{\partial y}=4\left(T-T_{h}\right) \int_{0}^{\infty} K_{\lambda_{h}}\left(\frac{\partial e_{\lambda p}}{\partial T}\right)_{h} \mathrm{~d} \lambda,
$$

where $K_{\lambda}$ is the absorption coefficient, $\lambda$ is the wave length, $e_{\lambda p}$ is the Plank's function and subscript ' $h$ ' indicates that all quantities have been evaluated at the temperature $T_{h}$ which is the temperature of the plate at time $t \leq 0$. Thus our study is limited to small difference of plate temperature to the fluid temperature.

On the use of the Equation (5), Equation (3) becomes

$$
\rho c_{p} \frac{\partial T}{\partial t}=k \frac{\partial^{2} T}{\partial y^{2}}-4\left(T-T_{h}\right) I+Q_{0}\left(T-T_{h}\right),
$$

where

$$
I=\int_{0}^{\infty} K_{\lambda_{h}}\left(\frac{\partial e_{\lambda p}}{\partial T}\right)_{h} d \lambda .
$$

Greif et al. [18] showed that, for an optically thin limit, the fluid does not absorb its own emitted radiation, this means that there is no self-absorption, but the fluid does absorb radiation emitted by the boundaries.

Using condition at $y=h$, Equation (2) yields

$$
-\frac{1}{\rho} \frac{\partial p}{\partial x}=0 \text {. }
$$

On the use of (8), Equation (2) becomes

$$
\frac{\partial u}{\partial t}=g \beta\left(T-T_{h}\right)+v \frac{\partial^{2} u}{\partial y^{2}}-\frac{\sigma B_{0}^{2}}{\rho} u .
$$

Introducing non-dimensional variables

$$
\eta=\frac{y}{h}, \quad \tau=\frac{v t}{h^{2}}, \quad u_{1}=\frac{u}{u_{0}}, \quad \theta=\frac{T-T_{h}}{T_{0}-T_{h}},
$$

Equations (9) and (6) become

$$
\begin{aligned}
& \frac{\partial u_{1}}{\partial \tau}=\frac{\partial^{2} u_{1}}{\partial \eta^{2}}+G r \theta-M^{2} u_{1}, \\
& \operatorname{Pr} \frac{\partial \theta}{\partial \tau}=\frac{\partial^{2} \theta}{\partial \eta^{2}}-R \theta+\phi \theta,
\end{aligned}
$$

where $M^{2}=\frac{\sigma B_{0}^{2} h^{2}}{\rho v}$ is the magnetic parameter, $R=\frac{4 I h^{2}}{k}$ the radiation parameter, $\phi=\frac{Q_{0} h^{2}}{k}$ the heat generation parameter, $G r=\frac{g \beta\left(T_{0}-T_{h}\right) h^{2}}{v u_{0}}$ the Grashof number and $\operatorname{Pr}=\frac{\rho v c_{p}}{k}$ the Prandtl number.

The corresponding initial and boundary conditions for $u_{1}$ and $\theta$ are

$$
\begin{array}{ll}
u_{1}=0, & \theta=0 \text { for } 0 \leq \eta \leq 1 \text { and } \tau \leq 0, \\
u_{1}=e^{a \tau}, & \theta=\tau \text { at } \eta=0 \text { for } \tau>0, \\
u_{1}=0, & \theta=0 \text { at } \eta=1 \text { for } \tau>0,
\end{array}
$$

where $a=\frac{a^{\prime} h^{2}}{v}$ is the accelerated parameter.

Taking Laplace transformation, the Equations (11) and (12) become

$$
\begin{aligned}
& s \bar{u}_{1}=\frac{\mathrm{d}^{2} \bar{u}_{1}}{\mathrm{~d} \eta^{2}}+G r \bar{\theta}-M^{2} \bar{u}_{1} \\
& \operatorname{Pr} s \bar{\theta}=\frac{\mathrm{d}^{2} \bar{\theta}}{\mathrm{d} \eta^{2}}-R \bar{\theta}+\phi \bar{\theta}
\end{aligned}
$$

where

$$
\begin{aligned}
& \bar{u}_{1}(\eta, s)=\int_{0}^{\infty} u_{1}(\eta, s) e^{-s \tau} \mathrm{d} \tau \\
& \text { and } \bar{\theta}(\eta, s)=\int_{0}^{\infty} \theta(\eta, s) e^{-s \tau} \mathrm{d} \tau .
\end{aligned}
$$

The corresponding boundary conditions for $\bar{u}_{1}$ and $\bar{\theta}$ are

$$
\begin{aligned}
& \bar{u}_{1}(0, s)=\frac{1}{s-a}, \quad \bar{\theta}(0, s)=\frac{1}{s^{2}}, \\
& \bar{u}_{1}(1, s)=0, \quad \bar{\theta}(1, s)=0 .
\end{aligned}
$$

The solutions of the Equations (15) and (14) subject to the boundary conditions (17) are easily obtained and are given by

$$
\begin{aligned}
& \bar{\theta}(\eta, s)=\frac{1}{s^{2}} \frac{\sinh \sqrt{\operatorname{Pr}(s+\alpha)}(1-\eta)}{\sinh \sqrt{\operatorname{Pr}(s+\alpha)}} \\
& \bar{u}_{1}(\eta, s)=\frac{1}{(s-a)} \frac{\sinh \sqrt{s+M^{2}}(1-\eta)}{\sinh \sqrt{s+M^{2}}} \\
& +\frac{G r}{(\operatorname{Pr}-1)(s+b) s^{2}} \\
& \times\left[\frac{\sinh \sqrt{s+M^{2}}(1-\eta)}{\sinh \sqrt{s+M^{2}}}-\frac{\sinh \sqrt{\operatorname{Pr}(s+\alpha)}(1-\eta)}{\sinh \sqrt{\operatorname{Pr}(s+\alpha)}}\right]
\end{aligned}
$$

where $\alpha=\frac{R-\phi}{P r}$ and $b=\frac{\alpha P r-M^{2}}{P r-1}$

The inverse transforms of (18) and (19) give the temperature and the velocity field distributions as

$$
\begin{aligned}
& \theta(\eta, \tau)=\sum_{n=0}^{\infty}\left[F_{1}(c \sqrt{\operatorname{Pr}}, \tau)-F_{1}(d \sqrt{\operatorname{Pr}}, \tau)\right] \\
& u_{1}(\eta, \tau)=\sum_{n=0}^{\infty}\left[\left\{F_{2}(c, \tau)-F_{2}(d, \tau)\right\}+\frac{G r}{(\operatorname{Pr}-1)}\right. \\
& \left.\times\left\{F_{3}(c, \tau)-F_{3}(d, \tau)-F_{4}(c \sqrt{\operatorname{Pr}}, \tau)+F_{4}(d \sqrt{\operatorname{Pr}}, \tau)\right\}\right]
\end{aligned}
$$


where $c=2 n+\eta, d=2 n+2-\eta$

$$
\begin{aligned}
F_{1}(z, \tau)= & \left(\frac{\tau}{2}+\frac{z}{4 \sqrt{\alpha}}\right) e^{z \sqrt{\alpha}} \operatorname{erfc}\left(\frac{z}{2 \sqrt{\tau}}+\sqrt{\alpha \tau}\right)+\left(\frac{\tau}{2}-\frac{z}{4 \sqrt{\alpha}}\right) e^{-z \sqrt{\alpha}} \operatorname{erfc}\left(\frac{z}{2 \sqrt{\tau}}-\sqrt{\alpha \tau}\right) \\
F_{2}(z, \tau)= & \frac{1}{2} e^{a \tau}\left[e^{z \sqrt{M^{2}+a}} \operatorname{erfc}\left(\frac{z}{2 \sqrt{\tau}}+\sqrt{\left(M^{2}+a\right) \tau}\right)+e^{-z \sqrt{M^{2}+a}} \operatorname{erfc}\left(\frac{z}{2 \sqrt{\tau}}-\sqrt{\left(M^{2}+a\right) \tau}\right)\right], \\
F_{3}(z, \tau)= & \frac{1}{b}\left[\left(\frac{\tau}{2}+\frac{z}{4 M}\right) e^{M z} \operatorname{erfc}\left(\frac{z}{2 \sqrt{\tau}}+M \sqrt{\tau}\right)+\left(\frac{\tau}{2}-\frac{z}{4 M}\right) e^{-M z} \operatorname{erfc}\left(\frac{z}{2 \sqrt{\tau}}-M \sqrt{\tau}\right)\right] \\
& -\frac{1}{2 b^{2}}\left[e^{M z} \operatorname{erfc}\left(\frac{z}{2 \sqrt{\tau}}+M \sqrt{\tau}\right)+e^{-M z} \operatorname{erfc}\left(\frac{z}{2 \sqrt{\tau}}-M \sqrt{\tau}\right)\right] \\
& +\frac{e^{-b \tau}}{2 b^{2}}\left[e^{z \sqrt{M^{2}-b}} \operatorname{erfc}\left(\frac{z}{2 \sqrt{\tau}}+\sqrt{\left(M^{2}-b\right) \tau}\right)+e^{-z \sqrt{M^{2}-b}} \operatorname{erfc}\left(\frac{z}{2 \sqrt{\tau}}-\sqrt{\left(M^{2}-b\right) \tau}\right)\right], \\
F_{4}(z, \tau)= & \frac{1}{b}\left[\left(\frac{\tau}{2}+\frac{z}{4 \sqrt{\alpha}}\right) e^{z \sqrt{\alpha}} \operatorname{erfc}\left(\frac{z}{2 \sqrt{\tau}}+\sqrt{\alpha \tau}\right)+\left(\frac{\tau}{2}-\frac{z}{4 \sqrt{\alpha}}\right) e^{-z \sqrt{\alpha}} \operatorname{erfc}\left(\frac{z}{2 \sqrt{\tau}}-\sqrt{\alpha \tau}\right)\right] \\
& -\frac{1}{2 b^{2}}\left[e^{z \sqrt{\alpha}} \operatorname{erfc}\left(\frac{z}{2 \sqrt{\tau}}+\sqrt{\alpha \tau}\right)+e^{-z \sqrt{\alpha}} \operatorname{erfc}\left(\frac{z}{2 \sqrt{\tau}}-\sqrt{\alpha \tau}\right)\right] \\
& +\frac{e^{-b \tau}}{2 b^{2}}\left[e^{z \sqrt{\alpha-b}} \operatorname{erfc}\left(\frac{z}{2 \sqrt{\tau}}+\sqrt{(\alpha-b) \tau}\right)+e^{-z \sqrt{\alpha-b}} \operatorname{erfc}\left(\frac{z}{2 \sqrt{\tau}}-\sqrt{(\alpha-b) \tau}\right)\right]
\end{aligned}
$$

and erfc is the complementary error function.

\subsection{Solution for Prandtl number $P r=1$}

As the Prandtl number is a measure of the relative importance of the viscosity and thermal conductivity of the fluid, the case $P r=1$ corresponds to those fluids whose momentum and thermal boundary layer thicknesses are of the same order of magnitude. Thus, the solution for the velocity field has to be re-derived when Prandtl number $\operatorname{Pr}=1$. The solution of the Equations (15) and (14) subject to the boundary conditions (17) are easily obtained and are given by

$$
\begin{gathered}
\bar{\theta}(\eta, s)=\frac{1}{s^{2}} \frac{\sinh \sqrt{s+\alpha}(1-\eta)}{\sinh \sqrt{s+\alpha}}, \\
\bar{u}_{1}(\eta, s)=\frac{1}{s-a} \frac{\sinh \sqrt{s+M^{2}}(1-\eta)}{\sinh \sqrt{s+M^{2}}} \\
+\frac{G r}{s^{2}\left(\alpha-M^{2}\right)}\left[\frac{\sinh \sqrt{s+M^{2}}(1-\eta)}{\sinh \sqrt{s+M^{2}}}-\frac{\sinh \sqrt{s+\alpha}(1-\eta)}{\sinh \sqrt{s+\alpha}}\right] .
\end{gathered}
$$

The inverse transforms of (23) and (24) give the temperature and velocity distributions as

$$
\theta(\eta, \tau)=\sum_{n=0}^{\infty}\left[F_{1}(c, \tau)-F_{1}(d, \tau)\right]
$$

$$
\begin{aligned}
u_{1}(\eta, \tau) & =\sum_{n=0}^{\infty}\left[\left\{F_{2}(c, \tau)-F_{2}(d, \tau)\right\}+\frac{G r}{\left(\alpha-M^{2}\right)}\right. \\
& \left.\times\left\{F_{5}(c, \tau)-F_{5}(d, \tau)-F_{1}(c, \tau)+F_{1}(d, \tau)\right\}\right] .
\end{aligned}
$$

where

$$
\begin{aligned}
F_{5}(z, \tau)= & \left(\frac{\tau}{2}+\frac{z}{4 M}\right) e^{M z} \operatorname{erfc}\left(\frac{z}{2 \sqrt{\tau}}+M \sqrt{\tau}\right) \\
& +\left(\frac{\tau}{2}-\frac{z}{4 M}\right) e^{-M z} \operatorname{erfc}\left(\frac{z}{2 \sqrt{\tau}}-M \sqrt{\tau}\right)
\end{aligned}
$$

and $F_{1}(z, \tau)$ and $F_{2}(z, \tau)$ are given by (22).

\section{Results and Discussion}

We have presented the non-dimensional velocity and temperature for several values of magnetic parameter $M^{2}$, radiation parameter $R$, heat generation parameter $\phi$, Prandtl number $\mathrm{Pr}$, Grashof number $\mathrm{Gr}$, accelerated parameter $a$ and time $\tau$ in Figures 2-12. Figures 2-8 represent the velocity $u_{1}$ against $\eta$ for several values of $M^{2}, R, \phi, P r, G r, a$, and $\tau$. It is seen from Figure 2 that the velocity $u_{1}$ decreases with an increase in magnetic parameter $M^{2}$. The application of the transverse magnetic field plays the role of a resistive type force (Lorentz force) similar to drag force (that acts in the opposite direction of the fluid 


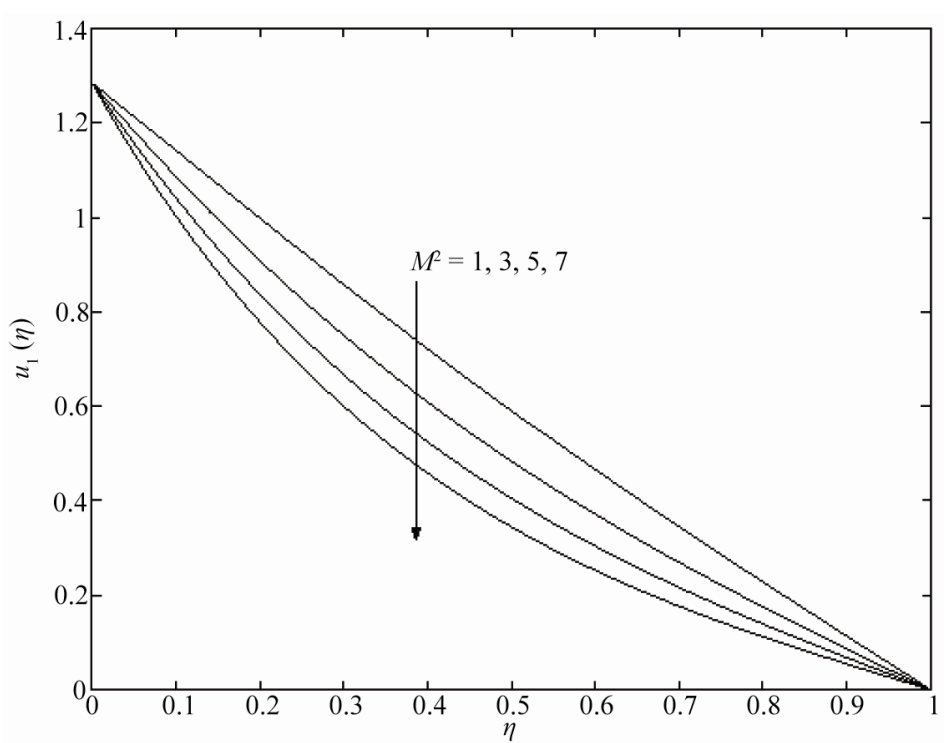

Figure 2. Velocity profiles for $M^{2}$ when $R=12, \phi=4, G r=5, P r=0.71, a=0.5$ and $\tau=0.5$.

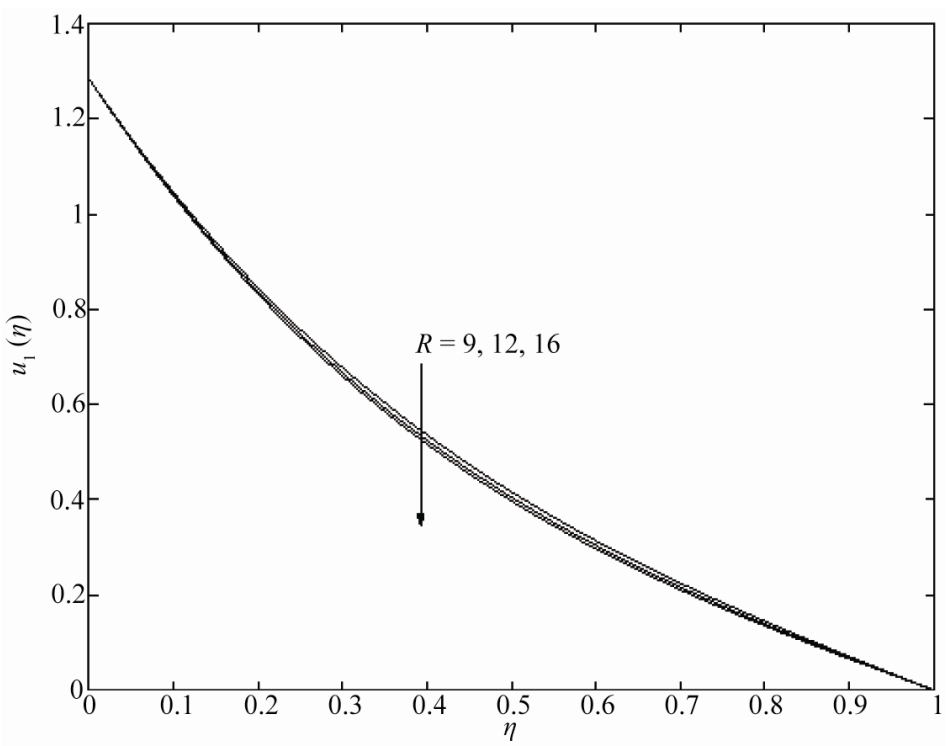

Figure 3. Velocity profiles for $R$ when $M^{2}=5, \phi=4, G r=5, P r=0.71, a=0.5$ and $\tau=0.5$.

motion) which tends to resist the flow thereby reducing its velocity. Figure 3 reveals that the velocity $u_{1}$ decreases with an increase in radiation parameter $R$. This shows that there is a fall in velocity in the presence of high radiation. It is seen from Figure 4 that the velocity $u_{1}$ increases with an increase in heat generation parameter $\phi$. As $\phi$ increases, heat generating capacity of the fluid increases which increases fluid temperature and hence the fluid velocity. Figure 5 shows that the velocity $u_{1}$ decreases with an increase in Prandtl number $\mathrm{Pr}$. Physically, this is true because the increase in the Prandtl number is due to increase in the viscosity of the fluid which makes the fluid thick and hence causes a decrease in the velocity of the fluid. It is observed from Figure 6 that an increase in $\mathrm{Gr}$ leads to rise in the values of velocity $u_{1}$. An increase in Grashof number leads to an increase in velocity, this is because, increase in Grashof number means more heating and less density. It is seen from Figures 7, 8 that the velocity $u_{1}$ increases with an increase in either accelerated parameter $a$ or time $\tau$. It is seen from Figure 9 that the temperature $\theta$ decreases as the radiation parameter $R$ increases. This result qualitatively agrees with expectations, since the effect of radiation is to decrease the rate of energy transport to the fluid, thereby decreasing the temperature of the fluid. It is seen from Figure 10 that the temperature $\theta$ increases as the heat generation parameter $\phi$ increases. This result agrees with expectations, as $\phi$ increases, heat genera- 


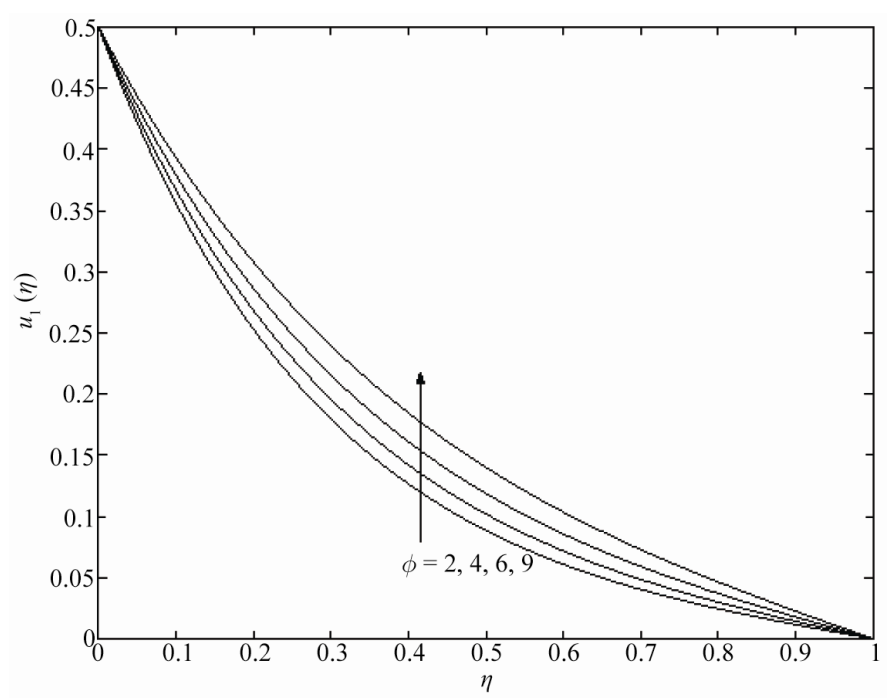

Figure 4. Velocity profiles for $\phi$ when $M^{2}=5, R=12, G r=5, P r=0.71, a=0.5$ and $\tau=0.5$.

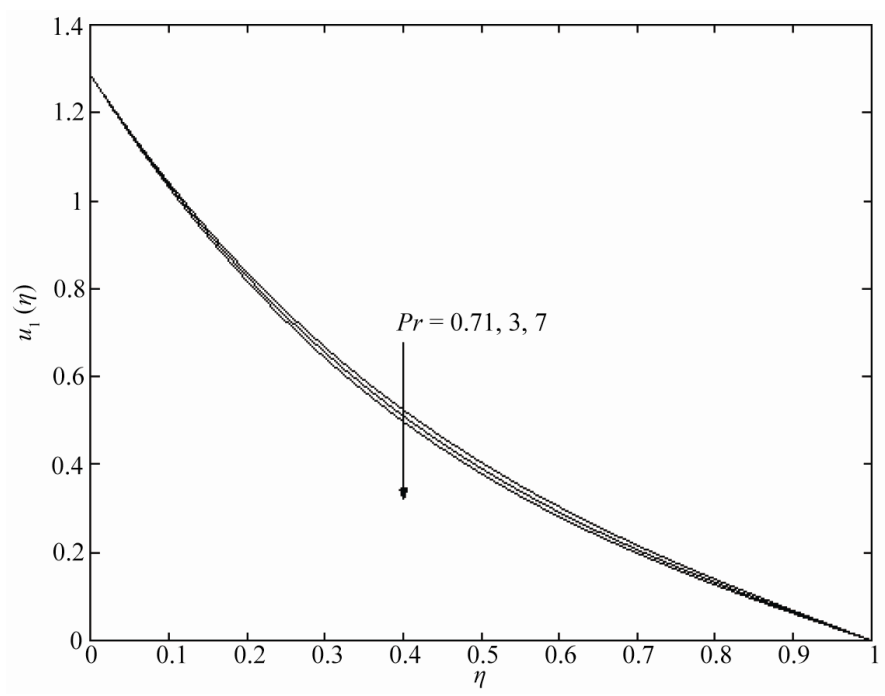

Figure 5. Velocity profiles for $\operatorname{Pr}$ when $M^{2}=5, R=12, G r=5, \phi=4, a=0.5$ and $\tau=0.5$.

ting capacity of the fluid increases and hence the fluid temperature increases. It is observed from Figure 11 that the temperature $\theta$ decreases with an increase in Prandtl number $\mathrm{Pr}$. This implies that an increase in Prandtl number leads to fall the thermal boundary layer flow. This is because fluids with large $P r$ have low thermal diffusivity which causes low heat penetration resulting in reduced thermal boundary layer. Figure $\mathbf{1 2}$ reveals that the temperature $\theta$ increases with an increase in time $\tau$. The trend shows that the temperature increases with increasing time. It is observed from Figures 9-12 that temperature decreases gradually from highest value on the moving plate to a zero value on the stationary plate.

The rate of heat transfer at the moving plate $(\eta=0)$ is given by

$$
\theta^{\prime}(0)=\left.\frac{\partial \theta}{\partial \eta}\right|_{\eta=0}==\sum_{n=0}^{\infty}\left[G_{1}\left(c^{\prime} \sqrt{\operatorname{Pr}}, \tau\right)-G_{1}\left(d^{\prime} \sqrt{\operatorname{Pr}}, \tau\right)\right],
$$

where $c^{\prime}=2 n, d^{\prime}=2 n+2$

$$
\begin{aligned}
G_{1}(z, \tau)= & \frac{\sqrt{\operatorname{Pr}}}{4 \sqrt{\alpha}}\left[e^{z \sqrt{\alpha}} \operatorname{erfc}\left(\frac{z}{2 \sqrt{\tau}}+\sqrt{\alpha \tau}\right)-e^{-z \sqrt{\alpha}} \operatorname{erfc}\left(\frac{z}{2 \sqrt{\tau}}-\sqrt{\alpha \tau}\right)\right] \\
& \left.+\sqrt{\alpha \operatorname{Pr}}\left[\left(\frac{\tau}{2}+\frac{z}{4 \sqrt{\alpha}}\right) e^{z \sqrt{\alpha}} \operatorname{erfc}\left(\frac{z}{2 \sqrt{\tau}}+\sqrt{\alpha \tau}\right)-\left(\frac{\tau}{2}-\frac{z}{4 \sqrt{\alpha}}\right) e^{-z \sqrt{\alpha}} \operatorname{erfc}\left(\frac{z}{2 \sqrt{\tau}}-\sqrt{\alpha \tau}\right)\right]-\sqrt{\frac{\operatorname{Pr} \tau}{\pi}} e^{-\left(\frac{z^{2}}{4 \tau}+\alpha \tau\right.}\right) .
\end{aligned}
$$




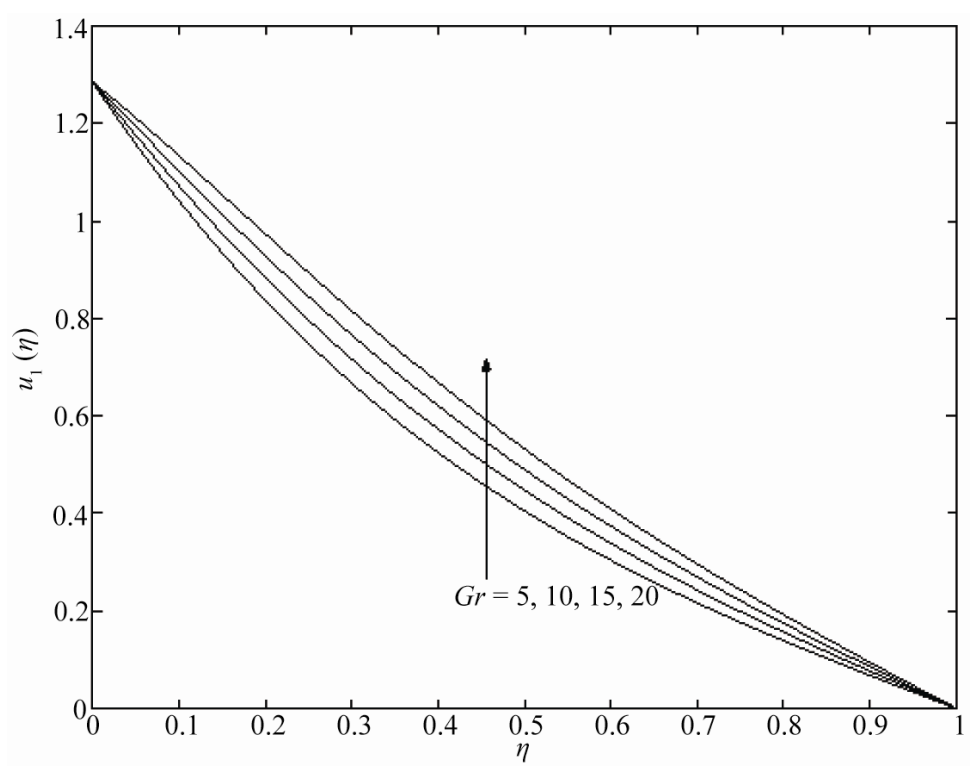

Figure 6. Velocity profiles for $G r$ when $M^{2}=5, R=12, \phi=4, P r=0.71, a=0.5$ and $\tau=0.5$.

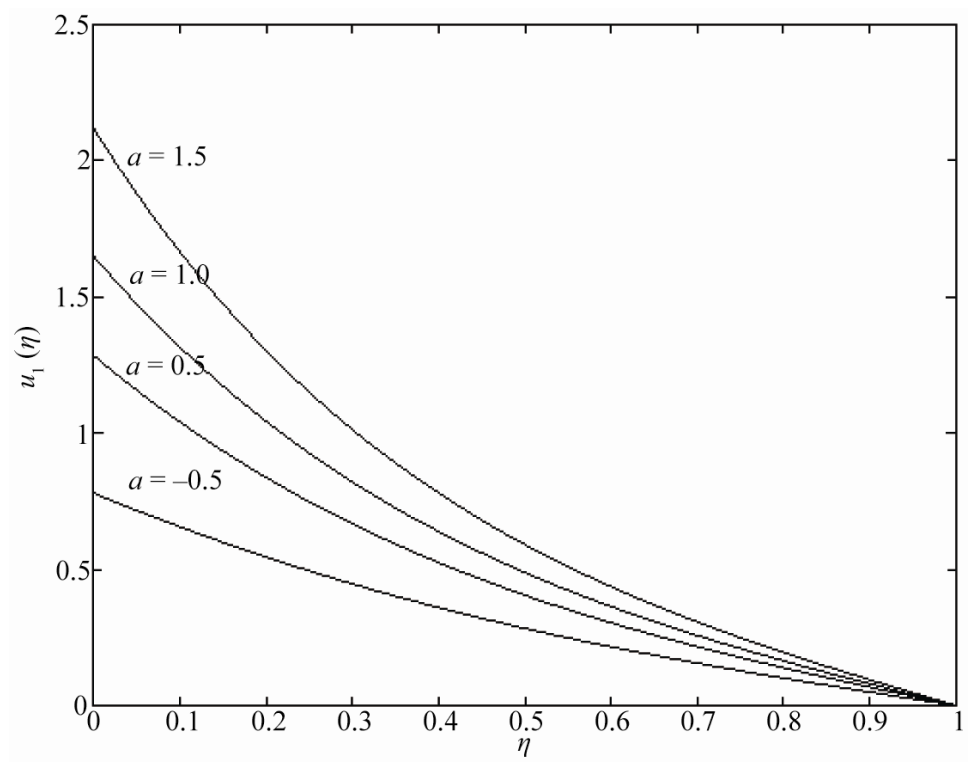

Figure 7. Velocity profiles for $a$ when $M^{2}=5, R=12, G r=5, \phi=4, \operatorname{Pr}=0.71$ and $\tau=0.5$.

Numerical results of the rate of heat transfer $-\theta^{\prime}(0)$ at the moving plate $(\eta=0)$ against the radiation parameter $R$ are presented in the Table 1 for various values of heat generation parameter $\phi$, Prandtl number $\operatorname{Pr}$ and time $\tau$. Table 1 shows that for the fixed value of radiation parameter $R$, the rate of heat transfer $-\theta^{\prime}(0)$ increases with an increase in either Prandtl number $\operatorname{Pr}$ or time $\tau$ and it decreases with an increase in heat generation parameter $\phi$. This may be explained by the fact that frictional forces become dominant with increasing values of $\mathrm{Pr}$ and hence yield greater heat transfer rates. Further, it is seen that for fixed value of $\phi, \operatorname{Pr}$ and $\tau$, the rate of heat transfer $-\theta^{\prime}(0)$ increases with an increase in radiation parameter.

From the physical point of view, it is necessary to know the shear stress at the moving plate. The nondimensional shear stress at the plate $(\eta=0)$ is obtained as follows:

$$
\tau_{x}=\left.\frac{\partial u_{1}}{\partial \eta}\right|_{\eta=0}=\sum_{n=0}^{\infty}\left[\left\{G_{2}\left(c^{\prime}, \tau\right)-G_{2}\left(d^{\prime}, \tau\right)\right\}+\frac{G r}{(\operatorname{Pr}-1)}\left\{G_{3}\left(c^{\prime}, \tau\right)-G_{3}\left(d^{\prime}, \tau\right)-G_{4}\left(c^{\prime} \sqrt{\operatorname{Pr}}, \tau\right)+G_{4}\left(d^{\prime} \sqrt{\operatorname{Pr}}, \tau\right)\right\} .\right.
$$

where 


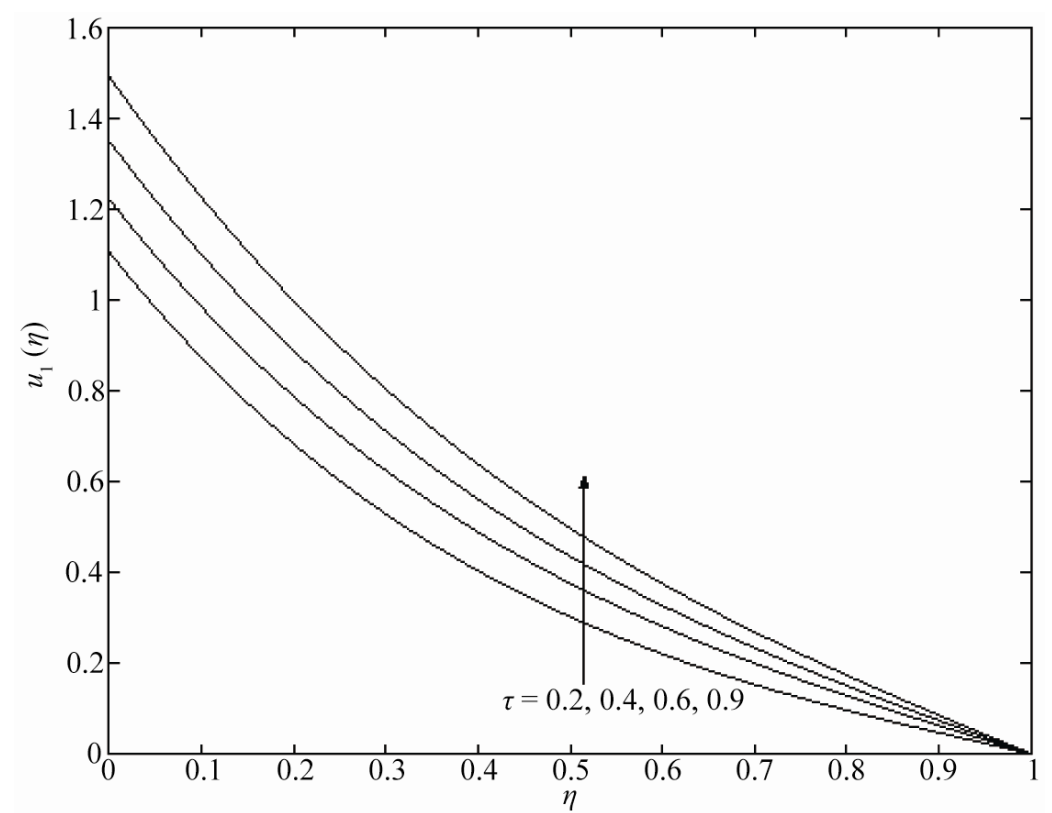

Figure 8. Velocity profiles for $\tau$ when $M^{2}=5, R=12, G r=5, \phi=4, a=0.5$ and $\operatorname{Pr}=0.71$.

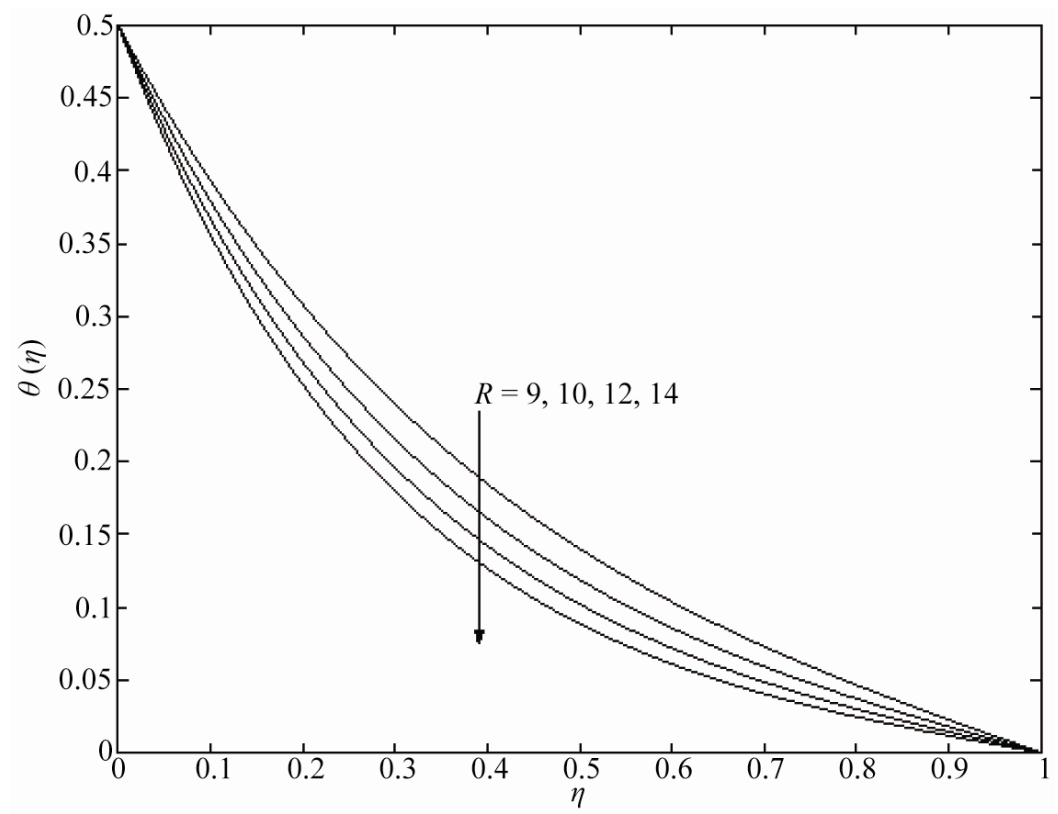

Figure 9. Temperature profiles for $R$ when $\operatorname{Pr}=0.71, \phi=4$ and $\tau=0.5$.

Table 1. Rate of heat transfer $-10^{-1} \theta^{\prime}(0)$ at the moving plate $\eta=0$ with $a=0.5$.

\begin{tabular}{|c|c|c|c|c|c|c|c|c|c|}
\hline \multirow{2}{*}{$\boldsymbol{R}$} & \multicolumn{3}{|c|}{$\phi$} & \multicolumn{3}{|c|}{$\mathrm{Pr}$} & \multicolumn{3}{|c|}{$\tau$} \\
\hline & 2 & 3 & 4 & 0.71 & 2 & 7 & 0.3 & 0.4 & 0.5 \\
\hline 8 & 1.36959 & 1.27657 & 1.17686 & 1.36959 & 1.62291 & 2.40040 & 0.87900 & 1.12448 & 1.36959 \\
\hline 10 & 1.53971 & 1.45702 & 1.36959 & 1.53971 & 1.76369 & 2.49186 & 0.97383 & 1.25683 & 1.53971 \\
\hline 12 & 1.69340 & 1.61833 & 1.53971 & 1.69340 & 1.89559 & 2.58105 & 1.06088 & 1.37716 & 1.69340 \\
\hline 14 & 1.83453 & 1.76535 & 1.69340 & 1.83453 & 2.01991 & 2.66810 & 1.14169 & 1.48812 & 1.83453 \\
\hline
\end{tabular}




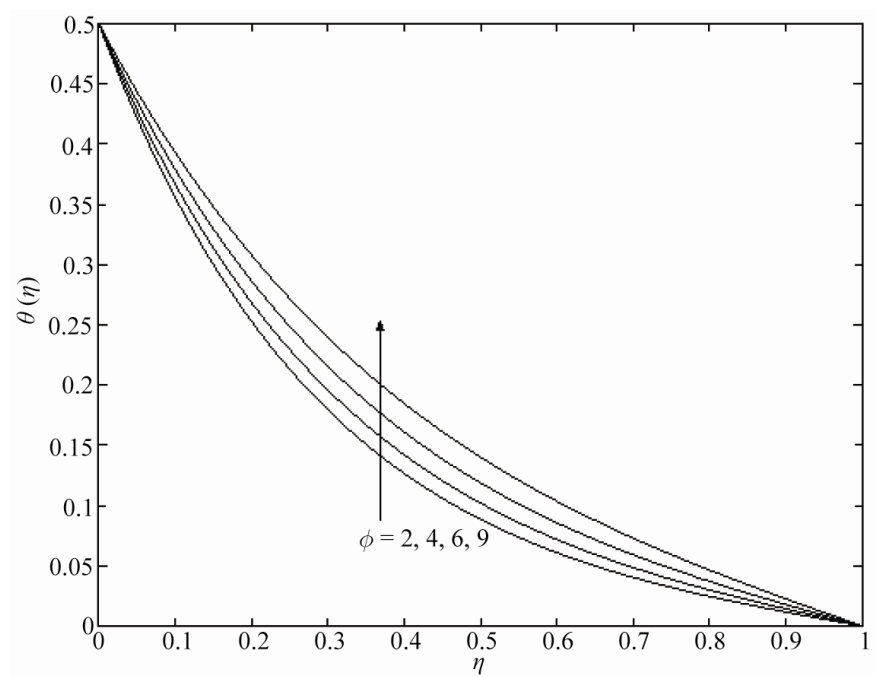

Figure 10. Temperature profiles for $\phi$ when $R=12, \operatorname{Pr}=0.71$ and $\tau=0.5$.

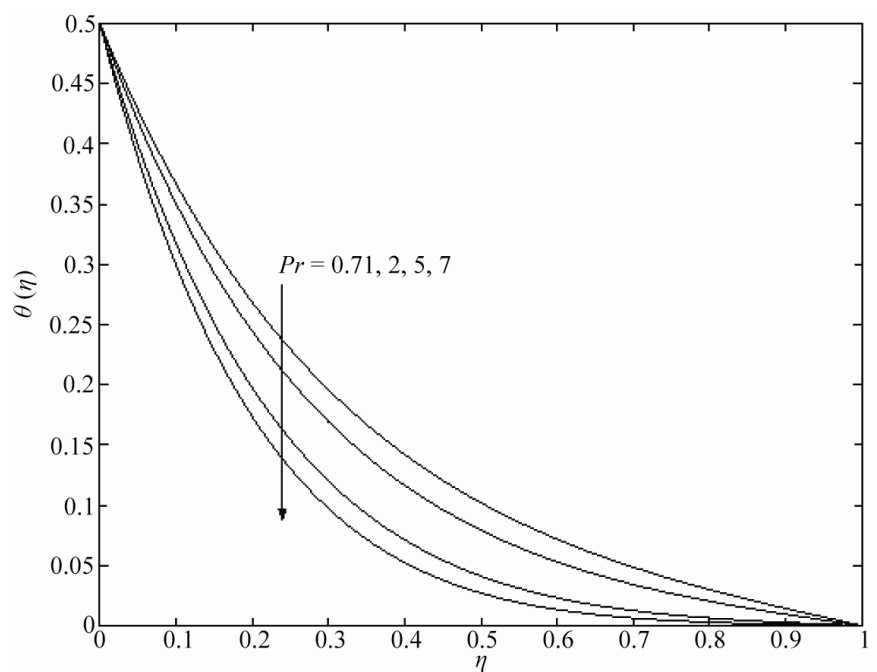

Figure 11. Temperature profiles for $\operatorname{Pr}$ when $R=12, \phi=4$ and $\tau=0.2$.

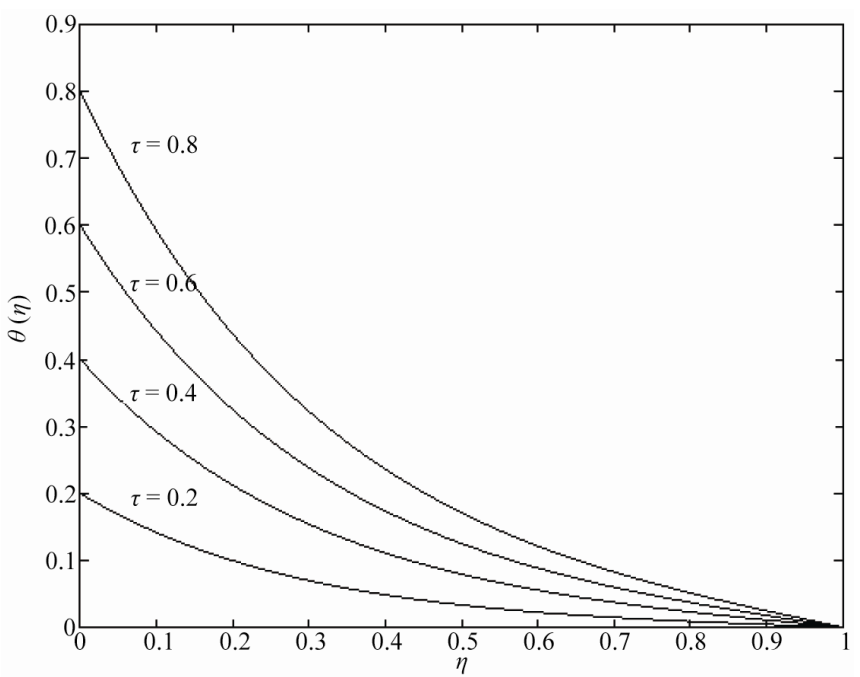

Figure 12. Temperature profiles for $\tau$ when $\operatorname{Pr}=0.71, R=12$ and $\phi=4$. 


$$
\begin{aligned}
& G_{2}(z, \tau) \\
& =\frac{1}{2} e^{a \tau}\left[\sqrt{M^{2}+a} \times\left\{e^{z \sqrt{M^{2}+a}} \operatorname{erfc}\left(\frac{Z}{2 \sqrt{\tau}}+\sqrt{\left(M^{2}+a\right) \tau}\right)-e^{-z \sqrt{M^{2}+a}} \operatorname{erfc}\left(\frac{Z}{2 \sqrt{\tau}}-\sqrt{\left(M^{2}+a\right) \tau}\right)\right\}-\frac{2}{\sqrt{\pi \tau}} e^{-\left\{\frac{z^{2}}{4 \tau}+\left(M^{2}+a\right) \tau\right\}}\right] \\
& G_{3}(z, \tau)=\left[\frac{1}{b}\left\{\frac{1}{4 M}+M\left(\frac{\tau}{2}+\frac{z}{4 M}\right)\right\}-\frac{M}{2 b^{2}}\right] \times e^{M z} \operatorname{erfc}\left(\frac{z}{2 \sqrt{\tau}}+M \sqrt{\tau}\right)-\left[\frac{1}{b}\left\{\frac{1}{4 M}+M\left(\frac{\tau}{2}-\frac{z}{4 M}\right)\right\}-\frac{M}{2 b^{2}}\right] \\
& \times e^{-M z} \operatorname{erfc}\left(\frac{z}{2 \sqrt{\tau}}-M \sqrt{\tau}\right)-\left(\frac{1}{b} \sqrt{\frac{\tau}{\pi}}-\frac{2}{b^{2} \sqrt{\pi \tau}}\right) e^{-\left(\frac{z^{2}}{4 \tau}+M^{2} \tau\right)} \\
& \left.+\frac{e^{-b \tau}}{2 b^{2}}\left[\sqrt{M^{2}-b} \times\left\{e^{z \sqrt{M^{2}-b}} \operatorname{erfc}\left(\frac{z}{2 \sqrt{\tau}}+\sqrt{\left(M^{2}-b\right) \tau}\right)-e^{-z \sqrt{M^{2}-b}} \operatorname{erfc}\left(\frac{z}{2 \sqrt{\tau}}-\sqrt{\left(M^{2}-b\right) \tau}\right)\right\}-\frac{2}{\sqrt{\pi \tau}} e^{-\left(\frac{z^{2}}{4 \tau}+\left(M^{2}-b\right) \tau\right.}\right)\right], \\
& G_{4}(z, \tau) \\
& =\left[\frac{1}{b}\left\{\frac{\sqrt{\operatorname{Pr}}}{4 \sqrt{\alpha}}+\sqrt{\alpha \operatorname{Pr}}\left(\frac{\tau}{2}+\frac{z}{4 \sqrt{\alpha}}\right)\right\}-\frac{\sqrt{\alpha \operatorname{Pr}}}{2 b^{2}}\right] \times e^{z \sqrt{\alpha}} \operatorname{erfc}\left(\frac{z}{2 \sqrt{\tau}}+\sqrt{\alpha \tau}\right)-\left[\frac{1}{b}\left\{\frac{\sqrt{\operatorname{Pr}}}{4 \sqrt{\alpha}}+\sqrt{\alpha \operatorname{Pr}}\left(\frac{\tau}{2}-\frac{z}{4 \sqrt{\alpha}}\right)\right\}-\frac{\sqrt{\alpha \operatorname{Pr}}}{2 b^{2}}\right] \\
& \times e^{-z \sqrt{\alpha}} \operatorname{erfc}\left(\frac{z}{2 \sqrt{\tau}}-\sqrt{\alpha \tau}\right) \\
& \left.+\frac{e^{-b \tau}}{2 b^{2}}\left[\sqrt{\operatorname{Pr}(\alpha-b)} \times\left\{e^{z \sqrt{\alpha-b}} \operatorname{erfc}\left(\frac{z}{2 \sqrt{\tau}}+\sqrt{(\alpha-b) \tau}\right)-e^{-z \sqrt{\alpha-b}} \operatorname{erfc}\left(\frac{z}{2 \sqrt{\tau}}-\sqrt{(\alpha-b) \tau}\right)\right\}-2 \sqrt{\frac{\operatorname{Pr}}{\pi \tau}} e^{-\left\{\frac{z^{2} \operatorname{Pr}}{4 \tau}+(\alpha-b) \tau\right.}\right\}\right] .
\end{aligned}
$$

For $\operatorname{Pr}=1$, the non-dimensional shear stress at the moving plate $(\eta=0)$ is given by

$$
\tau_{x}=\sum_{n=0}^{\infty}\left[\left\{G_{2}\left(c^{\prime}, \tau\right)-G_{2}\left(d^{\prime}, \tau\right)\right\}+\frac{G r}{\left(\alpha-M^{2}\right)}\left\{G_{5}\left(c^{\prime}, \tau\right)-G_{5}\left(d^{\prime}, \tau\right)-G_{6}\left(c^{\prime}, \tau\right)+G_{6}\left(d^{\prime}, \tau\right)\right\}\right]
$$

where

$$
\begin{aligned}
& G_{5}(z, \tau)=\left[\frac{1}{4 M}+M\left(\frac{\tau}{2}+\frac{z}{4 M}\right)\right] \\
& \quad \times e^{M z} \operatorname{erfc}\left(\frac{z}{2 \sqrt{\tau}}+M \sqrt{\tau}\right)-\left[\frac{1}{4 M}+M\left(\frac{\tau}{2}-\frac{z}{4 M}\right)\right] \\
& \left.\quad \times e^{-M z} \operatorname{erfc}\left(\frac{z}{2 \sqrt{\tau}}-M \sqrt{\tau}\right)-\sqrt{\frac{\tau}{\pi}} e^{-\left(\frac{z^{2}}{4 \tau}+M^{2} \tau\right.}\right),
\end{aligned}
$$

and $G_{1}(z, \tau)$ is given by (29)

$$
\begin{aligned}
& G_{6}(z, \tau) \\
= & {\left[\frac{1}{4 \sqrt{\alpha}}+\sqrt{\alpha}\left(\frac{\tau}{2}+\frac{z}{4 \sqrt{\alpha}}\right)\right] e^{z \sqrt{\alpha}} \operatorname{erfc}\left(\frac{z}{2 \sqrt{\tau}}+\sqrt{\alpha \tau}\right) } \\
& -\left[\frac{1}{4 \sqrt{\alpha}}+\sqrt{\alpha}\left(\frac{\tau}{2}-\frac{z}{4 \sqrt{\alpha}}\right)\right] e^{-z \sqrt{\alpha}} \operatorname{erfc}\left(\frac{z}{2 \sqrt{\tau}}-\sqrt{\alpha \tau}\right) \\
& \left.-\sqrt{\frac{\tau}{\pi}} e^{-\left(\frac{z^{2}}{4 \tau}+\alpha \tau\right.}\right) .
\end{aligned}
$$

and $G_{2}(z, \tau), G_{3}(z, \tau)$ and $G_{4}(z, \tau)$ are given by
(31).

Numerical results of the non-dimensional shear stress $\tau_{x}$ at the moving plate $(\eta=0)$ are presented in Figures 13-18 against Grashof number $G r$ for various values of magnetic parameter $M^{2}$, radiation parameter $R$, heat generation parameter $\phi$, Prandtl number $\operatorname{Pr}$ and time $\tau$. Figures 13 and 14 show that for the fixed value of Grashof number $G r$, the shear stress $\tau_{x}$ decreases with an increase in either magnetic parameter $M^{2}$ or radiation parameter $R$ and it increases with an increase in Grashof number $G r$ for the fixed values of $M^{2}$ and $R$. These results are in agreement with the fact that the velocity increases with an increase in $G r$ while it decreases with an increases in either $M^{2}$ or $R$. It is seen from Figures $\mathbf{1 5}$ and $\mathbf{1 6}$ that the shear stress $\tau_{x}$ increases with an increase in either heat generation parameter $\phi$ or Prandtl number $\operatorname{Pr}$ for fixed value of Grashof number Gr. Figures $\mathbf{1 7}$ and $\mathbf{1 8}$ reveal that the shear stress $\tau_{x}$ decreases with an increase in either accelerated parameter $a$ or time $\tau$ for fixed value of Grashof number $\mathrm{Gr}$ 


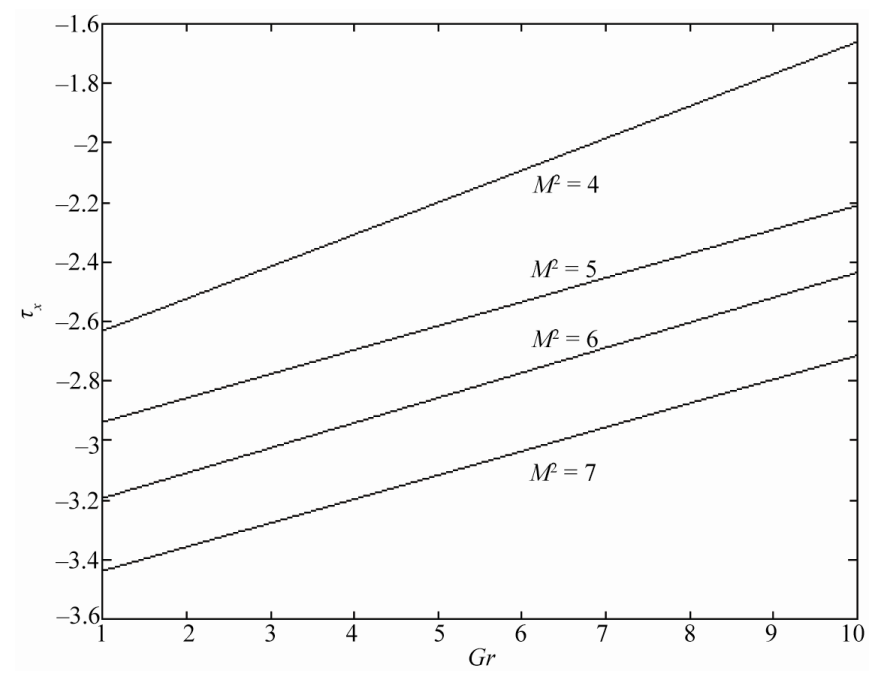

Figure 13. Shear stress $\tau_{x}$ for $M^{2}$ when $R=12, \phi=4, \operatorname{Pr}=0.71, a=0.5$ and $\tau=0.5$.

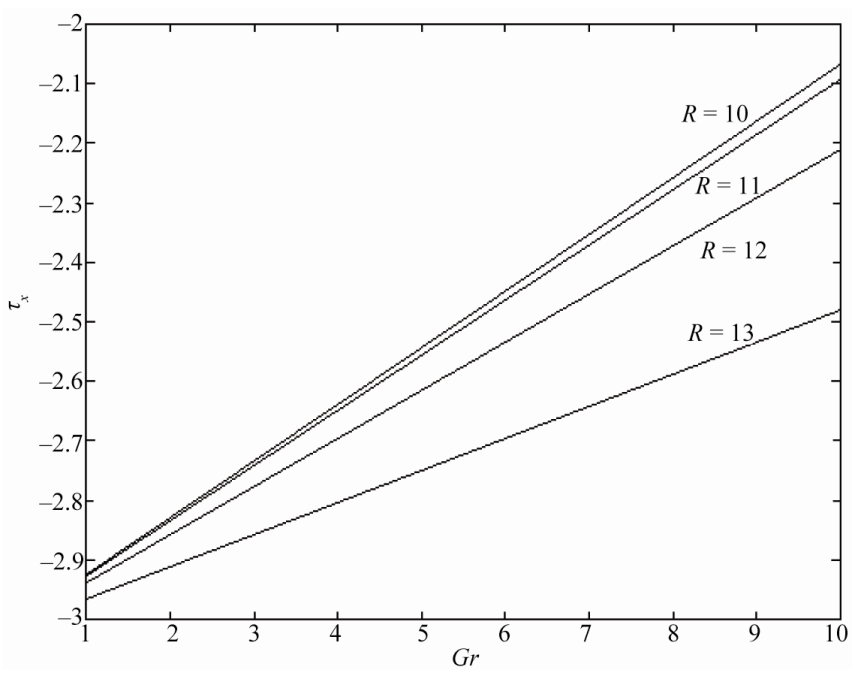

Figure 14. Shear stress $\tau_{x}$ for $R$ when $M^{2}=5, \phi=2, P r=0.71, a=0.5$ and $\tau=0.5$.

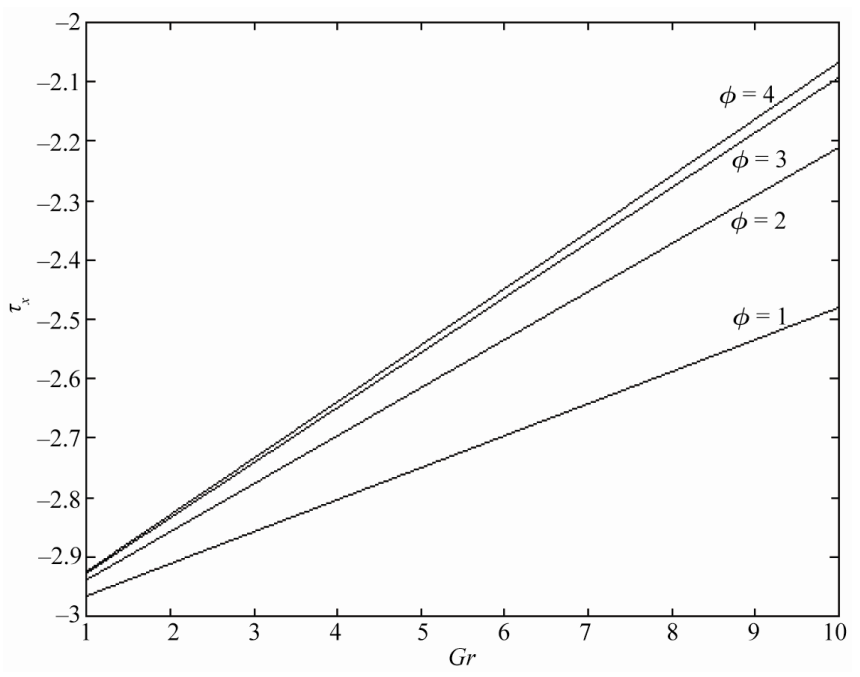

Figure 15. Shear stress $\tau_{x}$ for $\phi$ when $M^{2}=5, R=12, P r=0.71, a=0.5$ and $\tau=0.5$. 


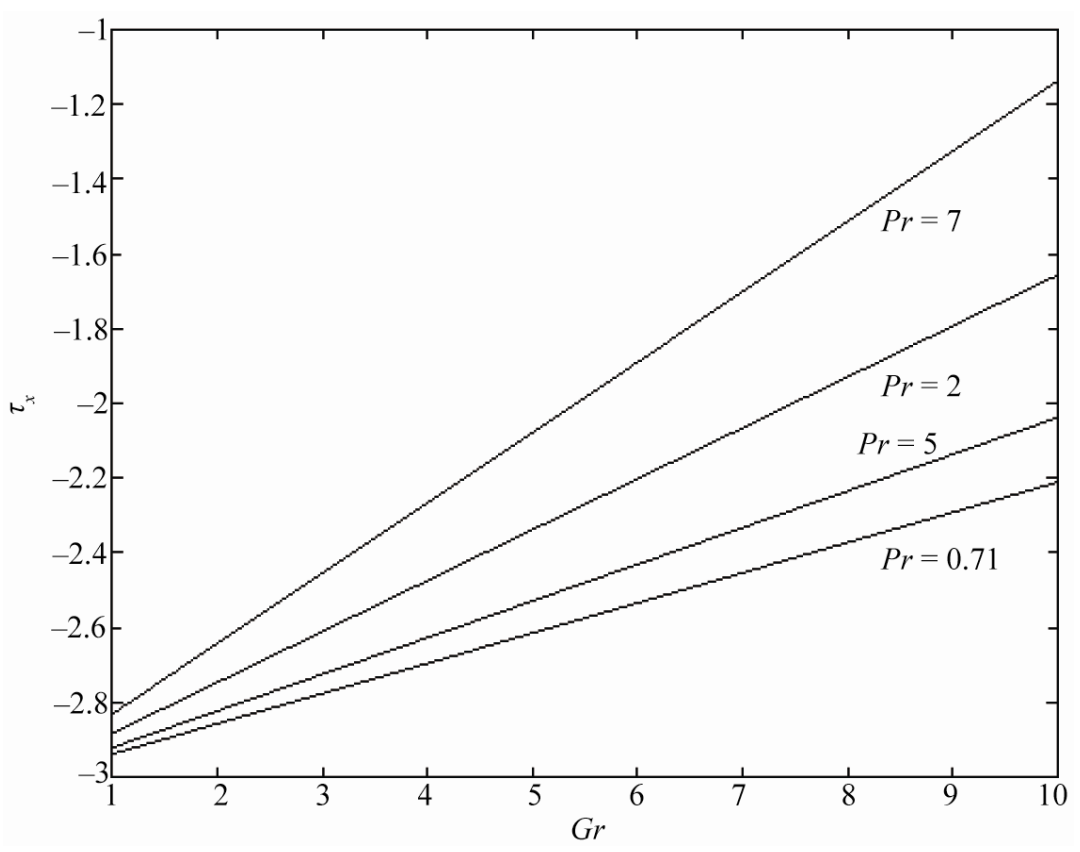

Figure 16. Shear stress $\tau_{x}$ for $\operatorname{Pr}$ when $M^{2}=5, R=12, \phi=4, a=0.5$ and $\tau=0.5$.

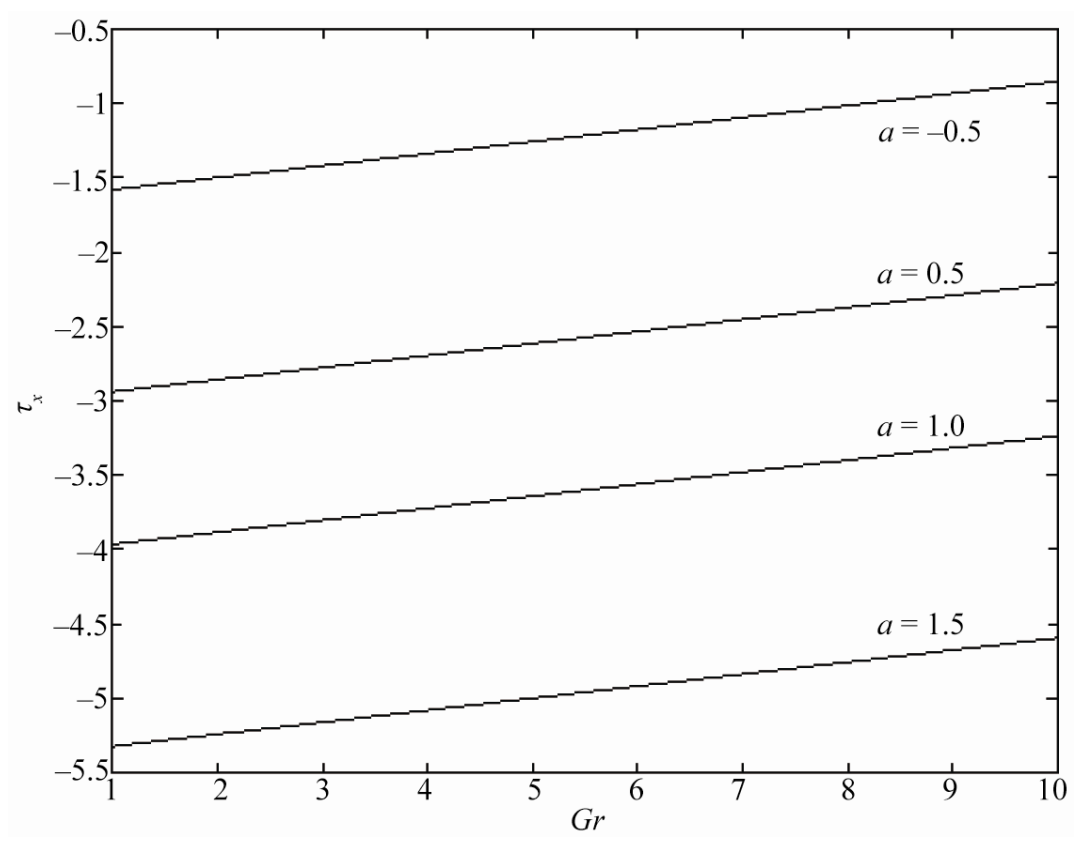

Figure 17. Shear stress $\tau_{x}$ for $a$ when $M^{2}=5, R=12, \phi=4, P r=0.71$ and $\tau=0.5$.

\section{Single Vertical Plate}

In the limit $h \rightarrow \infty$, that is, when one of the plates $(\eta=1)$ is placed at an infinite distance, then the problem is reduced to the flow past a vertical plate started exponentially accelerated with variable temperature in the presence of heat generation. In this case, on taking limits $d \rightarrow 0, n \rightarrow 0$ and $c \rightarrow \eta$, the Equations (20) and (21) become

$$
\theta(\eta, \tau)=F_{1}(\eta \sqrt{P r}, \tau)
$$




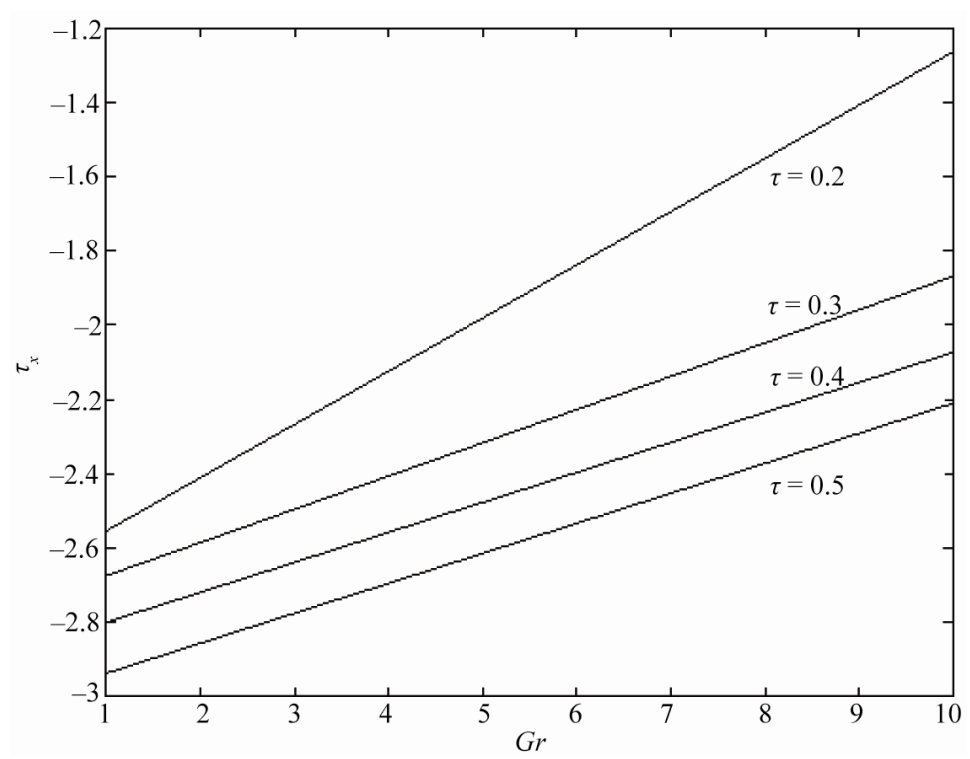

Figure 18. Shear stress $\tau_{x}$ for $\tau$ when $M^{2}=5, R=12, \phi=4, a=0.5$ and $\operatorname{Pr}=0.71$.

where

$$
\begin{aligned}
F_{1}(z, \tau)= & \left.\frac{\tau}{2}+\frac{z}{4 \sqrt{\alpha}}\right) e^{z \sqrt{\alpha}} \operatorname{erfc}\left(\frac{z}{2 \sqrt{\tau}}+\sqrt{\alpha \tau}\right)+\left(\frac{\tau}{2}-\frac{z}{4 \sqrt{\alpha}}\right) e^{-z \sqrt{\alpha}} \operatorname{erfc}\left(\frac{z}{2 \sqrt{\tau}}-\sqrt{\alpha \tau}\right), \\
F_{2}(z, \tau)= & \frac{1}{2} e^{a \tau}\left[e^{z \sqrt{M^{2}+a}} \operatorname{erfc}\left(\frac{z}{2 \sqrt{\tau}}+\sqrt{\left(M^{2}+a\right) \tau}\right)+e^{-z \sqrt{M^{2}+a}} \operatorname{erfc}\left(\frac{z}{2 \sqrt{\tau}}-\sqrt{\left(M^{2}+a\right) \tau}\right)\right], \\
F_{3}(z, \tau)= & \frac{1}{b}\left[\left(\frac{\tau}{2}+\frac{z}{4 M}\right) e^{M z} \operatorname{erfc}\left(\frac{z}{2 \sqrt{\tau}}+M \sqrt{\tau}\right)+\left(\frac{\tau}{2}-\frac{z}{4 M}\right) e^{-M z} \operatorname{erfc}\left(\frac{z}{2 \sqrt{\tau}}-M \sqrt{\tau}\right)\right] \\
& -\frac{1}{2 b^{2}}\left[e^{M z} \operatorname{erfc}\left(\frac{z}{2 \sqrt{\tau}}+M \sqrt{\tau}\right)+e^{-M z} \operatorname{erfc}\left(\frac{z}{2 \sqrt{\tau}}-M \sqrt{\tau}\right)\right] \\
& +\frac{e^{-b \tau}}{2 b^{2}}\left[e^{z \sqrt{M^{2}-b}} \operatorname{erfc}\left(\frac{z}{2 \sqrt{\tau}}+\sqrt{\left(M^{2}-b\right) \tau}\right)+e^{-z \sqrt{M^{2}-b}} \operatorname{erfc}\left(\frac{z}{2 \sqrt{\tau}}-\sqrt{\left(M^{2}-b\right) \tau}\right)\right], \\
F_{4}(z, \tau)= & \frac{1}{b}\left[\left(\frac{\tau}{2}+\frac{z}{4 \sqrt{\alpha}}\right) e^{z \sqrt{\alpha}} \operatorname{erfc}\left(\frac{z}{2 \sqrt{\tau}}+\sqrt{\alpha \tau}\right)+\left(\frac{\tau}{2}-\frac{z}{4 \sqrt{\alpha}}\right) e^{-z \sqrt{\alpha}} \operatorname{erfc}\left(\frac{z}{2 \sqrt{\tau}}-\sqrt{\alpha \tau}\right)\right] \\
& -\frac{1}{2 b^{2}}\left[e^{z \sqrt{\alpha}} \operatorname{erfc}\left(\frac{z}{2 \sqrt{\tau}}+\sqrt{\alpha \tau}\right)+e^{-z \sqrt{\alpha}} \operatorname{erfc}\left(\frac{z}{2 \sqrt{\tau}}-\sqrt{\alpha \tau}\right)\right] \\
& +\frac{e^{-b \tau}}{2 b^{2}}\left[e^{z \sqrt{\alpha-b}} \operatorname{erfc}\left(\frac{z}{2 \sqrt{\tau}}+\sqrt{(\alpha-b) \tau}\right)+e^{-z \sqrt{\alpha-b}} \operatorname{erfc}\left(\frac{z}{2 \sqrt{\tau}}-\sqrt{(\alpha-b) \tau}\right)\right]
\end{aligned}
$$

and

$$
\begin{aligned}
& \eta=\frac{u_{0} y}{v}, \tau=\frac{t}{t_{0}}, t_{0}=\frac{v}{u_{0}^{2}}, u_{1}=\frac{u}{u_{0}}, \theta=\frac{T-T_{h}}{T_{0}-T_{h}}, \\
& M^{2}=\frac{\sigma B_{0}^{2} v}{\rho u_{0}^{2}}, R=\frac{4 I v^{2}}{k u_{0}^{2}}, \phi=\frac{Q_{0} v^{2}}{k u_{0}^{2}}, \\
& G r=\frac{g \beta v\left(T_{0}-T_{h}\right)}{u_{0}^{3}}, \operatorname{Pr}=\frac{\mu c_{p}}{k} .
\end{aligned}
$$

Equations (34) and (35) are identical with the Equa- tions (9) and (10) of Kumar and Varma [17]. For $\operatorname{Pr}=1$, the solutions (25) and (26) for the temperature and velocity distributions yield

$$
\begin{gathered}
\theta(\eta, \tau)=F_{1}(\eta, \tau), \\
u_{1}(\eta, \tau)=F_{2}(\eta, \tau)+\frac{G r}{\left(\alpha-M^{2}\right)}\left[F_{5}(\eta, \tau)-F_{1}(\eta, \tau)\right],
\end{gathered}
$$

where $F_{1}(z, \tau), F_{2}(z, \tau)$ and $F_{3}(z, \tau)$ are given by (36) and $F_{5}(z, \tau)$ is given by (27). 


\section{Conclusion}

The radiation effects on free convection MHD Couette flow started exponentially accelerated with variable temperature in the presence of heat generation have been studied. The dimensionless governing equations are solved by the usual Laplace transform technique.It is observed that the velocity $u_{1}$ decreases with an increase in either magnetic parameter $M^{2}$ or radiation parameter $R$ or Prandtl number $\mathrm{Pr}$. It is also observed that the velocity $u_{1}$ increases with an increase in either heat generation parameter $\phi$ or Grashof number $G r$ or accelerated parameter $a$ or time $\tau$. An increase in either radiation parameter $R$ or Prandtl number $P r$ leads to fall in the fluid temperature $\theta$. It is seen that the fluid temperature $\theta$ increases with an increase in either heat generation parameter $\phi$ or time. Further, it is seen that the shear stress at the moving plate $\tau_{x}$ decreases with an increase in either $M^{2}$ or radiation parameter $R$ while it increases with an increase in either heat generation parameter $\phi$ or Prandtl number $\mathrm{Pr}$. The rate of heat transfer $-\theta^{\prime}(0)$ increases with an increase in either Prandtl number $\operatorname{Pr}$ or time $\tau$ whereas it decreases with an increase in heat generation parameter $\phi$.

\section{REFERENCES}

[1] A. K. Singh, "Natural Convection in Unsteady Couette Motion,” Defense Science Journal, Vol. 38, No. 1, 1988, pp. $35-41$.

[2] A. K. Singh and T. Paul, "Transient Natural Convection between Two Vertical Walls Heated/Cooled Asymetrically," International Journal of Applied Mechanics and Engineering, Vol. 11, No. 1, 2006, pp. 143-154.

[3] B. K. Jha, A. K. Singh and H. S. Takhar, "Transient Free Convection Flow in a Vertical Channel due to Symmetric Heating," International Journal of Applied Mechanics and Engineering, Vol. 8, No. 3, 2003, pp. 497-502.

[4] H. M. Joshi, "Transient Effects in Natural Convection Cooling of Vertical Parallel Plates," International Communications in Heat and Mass Transfer, Vol. 15, No. 2, 1988, pp. 227-238. doi:10.1016/0735-1933(88)90068-1

[5] O. Miyatake and T. Fujii, "Free Convection Heat Transfer between Vertical Plates-One Plate Isothermally Heated and Other Thermally Insulated," Heat Transfer-Japanese Research, Vol. 1, 1972, pp. 30-38.

[6] H. Tanaka, O. Miyatake, T. Fujii and M. Fujii, "Natural Convection Heat Transfer between Vertical Parallel Plates-One Plate with a Uniform Heat Flux and the Other Thermally Insulated," Heat Transfer-Japanese Research, Vol. 2, 1973, pp. 25-33.

[7] H. K. Mohanty, "Transient Free Convection Horizontal
Laminar Flow between Two Parallel Plates," Acta Mechanica, Vol. 15, No. 3-4, 1972, pp. 273-293.

doi:10.1007/BF01304296

[8] B. K. Jha, "Natural Convection in Unsteady MHD Couette Flow," Heat and Mass Transfer, Vol. 37, No. 4-5, 2001, pp. 329-331. doi:10.1007/PL00013295

[9] A. Ogulu and S. Motsa, "Radiative Heat Transfer to Magnetohydrodynamic Couette Flow with Variable Wall Temperature," Physica Scripta, Vol. 71, No. 4, 2005, pp. 336-339. doi:10.1238/Physica.Regular.071a00336

[10] R. C. Chaudhary and P. Jain, "Exact Solutions of Incompressible Couette Flow with Constant Temperature and Constant Heat Flux on Walls in the Presence of Radiation," Turkish Journal of Engineering \& Environmental Sciences, Vol. 3, No. 1, 2007, pp. 297-304.

[11] P. Mebine, "Radiation Effects on MHD Couette Flow With Heat Transfer between Two Parallel Plates," Global Journal of Pure and Applied Mathematics, Vol. 3, No. 2, 2007, pp. 1-12.

[12] B. K. Jha and A. O. Ajibade, "Free Convective Flow of Heat Generating/Absorbing Fluid between Vertical Porous Plates with Periodic Heat Input," International Communications in Heat and Mass Transfer, Vol. 36, No. 6, 2009, pp. 624-631. doi:10.1016/j.icheatmasstransfer.2009.03.003

[13] B. K. Jha and A. O. Ajibade, "Unsteady Free Convective Couette Flow of Heat Generating/Absorbing Fluid," International Journal of Energy and Technology, Vol. 2, No. 12, 2010, pp. 1-9.

[14] C. Israel-Cookey, E. Amos and C. Nwaigwe, "MHD Oscillatory Couette Flow of a Radiating Viscous Fluid in a Porous Medium with Periodic Wall Temperature," American Journal of Scientific Industrial Research, Vol. 1, No. 2, 2010, pp. 326-331.

[15] M. Narahari, "Effects Of Thermal Radiation and Free Convection Currents on the Unsteady Couette Flow between Two Vertical Parallel Plates with Constant Heat Flux at one Boundary," WSEAS Transactions on Heat and Mass Transfer, Vol. 5, No. 1, 2010, pp. 21-30.

[16] R. K. Deka and A. Bhattacharya, "Unsteady Free Convective Couette Flow of Heat Generating/Absorbing Fluid in Porous Medium," International Journal of Mathematical Archive, Vol. 2, No. 6, 2011, pp. 853-863.

[17] A. G. V. Kumar and S. V. K. Varma, "Radiation Effects on MHD Flow Past an Impulsively Started Exponentially Accelerated Vertical Plate with Variable Temperature in the Presence of Heat Generation," International Journal of Engineering Science and Technology, Vol. 3, No. 4, 2011, pp. 2897-2909.

[18] R. Grief, I. S. Habib and J. C. Lin, "Laminar Convection of a Radiating Gas in a Vertical Channel," Journal of Fluid Mechanics, Vol. 46, 1970, pp. 513-520. doi:10.1017/S0022112071000673 